\title{
Change in the Shape of Dendritic Spines Caused by Overexpression of Drebrin in Cultured Cortical Neurons
}

\author{
Kensuke Hayashi and Tomoaki Shirao \\ Department of Neurobiology and Behavior, Gunma University School of Medicine, 3-39-22 Showamachi, \\ Maebashi, 371, Japan
}

Dendritic spines are known to be extremely motile, providing a structural mechanism for synaptic plasticity. Actin filaments are thought to be responsible for the changes in the shape of spines. We tested our hypothesis that drebrin, an actin-binding protein, is a regulator of spine shape. In high-density long-term primary cultures of rat cerebral cortex neurons, drebrin was colocalized with actin filaments at spines. We introduced drebrin tagged with green fluorescent protein (GFP) into these neurons to test the ability of exogenous drebrin to localize at spines and the effect of overexpression of drebrin on spine shape. We observed that exogenous drebrin indeed accumulated in spines. But when the actin-binding domain of drebrin was deleted, the protein was distributed in both spines and

Many reports have appeared on dendritic spines changing their shape during neuronal development, as well as in the adult brain in response to various kind of stimuli (for review, see Koch and Zador, 1993). Recent studies of living neurons have provided direct evidence for the highly motile nature of spines formerly studied in static images from fixed neurons. Observation of fluorescently labeled spines in living hippocampal CA1 neurons in brain slices revealed the change in their morphology after chemical induction of long-term potentiation (LTP) (Hosokawa et al., 1995). A video recording of the morphology of spines was made from cultured cortical neurons expressing green fluorescent protein (GFP)-tagged actin. It demonstrated changes in their shape within seconds (Fischer et al., 1998).

The change in spine shape is an especially noteworthy phenomenon because it may account for the plasticity of synaptic transmission (for review, see Koch and Zador, 1993; Harris and Kater, 1994). The change in spine shape may alter the peak concentration of $\mathrm{Ca}^{2+}$ within spines and influence the efficiency of $\mathrm{Ca}^{2+}$ dependent enzymatic reactions associated with the development of LTP (Gold and Bear, 1994). When the change in spine shape results in the perforation or split of the postsynaptic density, two functionally separated active zones are created, and then synaptic transmission is augmented (Edwards, 1995).

Dendritic spines are extremely enriched with actin filaments

Received Oct. 5, 1998; revised Feb. 26, 1999; accepted March 3, 1999.

This research was supported in part by Grants-in-Aid (07279107, 09480219, 09780717 and 09280205 for Scientific Research on Priority Areas) from the Ministry of Education, Science, and Culture of Japan. We thank Shinobu C. Fujita at Mitsubishi Kagaku Lifescience Institute (Japan) for providing us with an excellent monoclonal antibody against green fluorescent protein. We also thank Erin Kinnally for her critical reading of this manuscript.

Correspondence should be addressed to Kensuke Hayashi, Laboratory of Molecular and Cellular Morphology, Institute for Molecular and Cellular Regulation, Gunma University, 3-39-15, Showamachi, Maebashi, Gunma 371-8512, Japan Copyright $(\odot 1999$ Society for Neuroscience $0270-6474 / 99 / 193918-08 \$ 05.00 / 0$ dendritic shafts, indicating that accumulation of drebrin in the spines required its actin-binding activity. Statistical analysis of the lengths of spines as determined from confocal laser microscopic images revealed that the spines were significantly longer in GFP-drebrin-expressing neurons than in GFP-expressing neurons. The longer spines labeled with GFP-drebrin were demonstrated to be postsynaptic by double labeling of the presynaptic terminals with antibody against synaptophysin. These results directly indicate that drebrin binds to actin filaments at dendritic spines and alters spine shape.

Key words: actin; spine; drebrin; plasticity; green fluorescent protein; primary culture

(Fifkova and Delay, 1982; Matus et al., 1982). These actin filaments are thought to be the primary modulators of the change of shape of spines because actin-depolymerizing agents suppressed the dynamic motility of spines (Fischer et al., 1998) and caused disappearance of spines (Allison et al., 1998). However, very little is known about the molecular mechanisms by which the motility of the actin filaments within spines are regulated.

We have hypothesized that drebrin, an actin-binding protein (for review, see Shirao, 1995), is one of the regulators of the motility of actin filaments during the change of shape of spines (Hayashi et al., 1996) based on the following evidence. (1) This actin-binding protein is localized at dendritic spines (Hayashi et al., 1996). (2) Because its affinity for actin is relatively high, actin binding of drebrin competes with other actin-binding proteins. Therefore, the arrangement and dynamics of actin filaments would be altered when drebrin predominates (Ishikawa et al., 1994; Sasaki et al., 1996). (3) A biochemical study revealed that drebrin inhibits the interaction between actin and myosin in vitro (Hayashi et al., 1996). This suggests that it possibly regulates contractility of actin filaments. (4) Introduction of drebrin into fibroblasts induced remodeling of actin filaments, causing a change in cell shape (Shirao et al., 1992, 1994).

The purpose of this study is to confirm our hypothesis by introducing excessive drebrin into primary cultures of cortical neurons and analyzing its effect on spine shape. We found that exogenous drebrin tagged with GFP localized at the spines and caused spines to lengthen. These results provide the first direct evidence for the involvement of actin-binding proteins in the regulation of spine shape.

\section{MATERIALS AND METHODS}

Primary cultures of cortical neurons. The cerebral cortices of 20-d-old fetal rats were dissociated by treatment with $9 \mathrm{U} / \mathrm{ml}$ papain (Worthington Biochemical, Lakewood, NJ) for 20 min, followed by trituration with a 
pipette. Dissociated cells were plated on polyethylenimine-coated cover glasses at a density of $2 \times 10^{6} / \mathrm{ml}$. Cells were cultured in MEM containing $6 \mathrm{gm} / 1$ glucose, $1 \mathrm{~mm}$ pyruvate, $5 \%$ horse serum, and $5 \%$ fetal bovine serum. On day $5, \mathrm{AraC}$ was added to reduce the proliferation of glial cells. Half of the medium was exchanged twice a week with medium that was conditioned with a confluent monolayer culture of astroglial cells for $24 \mathrm{hr}$. Transfection of neurons with cDNAs was performed on day 7, using Transfectum (Biosepra, Marlborough, MA). Three weeks after plating, the cells were fixed with $4 \%$ paraformaldehyde in $0.1 \mathrm{M}$ phosphate buffer, $\mathrm{pH} 7.2$, and observed. DiI-labeling was performed by adding fine grains of DiI suspended in PBS onto the fixed culture.

Chinese hamster ovary cells. Chinese hamster ovary (CHO)-K1 cells were cultured in Ham's F-12 nutrient mixture supplemented with $10 \%$ FBS. Transfection was performed with Tfx-20 (Promega, Madison, WI). Three days after transfection, the cells were fixed and permeabilized with $0.1 \%$ Triton X-100 in PBS for 15 min. Actin filaments were labeled by incubating the cells with rhodamine-phalloidin (Molecular Probes, Eugene, OR) for $30 \mathrm{~min}$. The fluorescence of GFP fusions was observed with a FITC filter set and that of rhodamine-phalloidin with a rhodamine filter set.

Immunocytochemistry. For double staining of drebrin and actin filaments in primary cultures, neurons at 3 weeks in vitro were fixed with $4 \%$

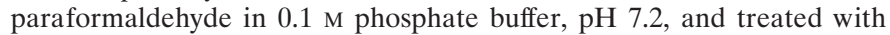
$0.1 \%$ Triton X-100 in PBS. They were incubated with $3 \%$ bovine serum albumin in PBS for $>1 \mathrm{hr}$ and then incubated with a monoclonal anti-drebrin antibody (M2F6; Medical and Biological Laboratories, Nagoya, Japan). After washing with PBS for $30 \mathrm{~min}$, they were incubated with the secondary antibody for $1 \mathrm{hr}$ and washed again for $30 \mathrm{~min}$. The secondary antibody was FITC-conjugated antibody against mouse IgG antibody (Tago, Camarillo, CA) and was used as a mixture with rhodamine-phalloidin (Molecular Probes).

For the staining of synaptophysin at GFP-drebrin-labeled spines, neurons transfected with GFP-drebrin cDNA were fixed and immunostained with a monoclonal anti-synaptophysin antibody (Obata et al., 1986) and rhodamine-conjugated antibodies against mouse IgG (Cappel, Durham, NC) using the same procedures described above.

cDNA constructs. Enhanced GFP (EGFP)-C1 vector (Clontech, Palo Alto, CA) was used to construct GFP-drebrin fusion cDNAs. This contains the enhanced GFP sequence with multiple point mutations, as well as codon optimization for mammalian expression. The expression is driven by a cytomegalovirus immediate early promoter. Drebrin cDNA inserts with or without the actin-binding sequence were generated by PCR using rat drebrin A cDNA (Shirao et al., 1992) as a template. The intact drebrin cDNA insert was amplified using a 5' primer (AATCTCGAGGCATGGCCGGCGTCATCTTC) that contains a cDNA sequence encoding the $\mathrm{N}$ terminus of drebrin and an additional $\mathrm{XhoI}$ site in frame with the GFP sequence at its $5^{\prime}$ end, and a $3^{\prime}$ primer (TTTGGATCCCCACCCTCGAAGCCCTCTTC) that contains an antisense sequence encoding the $\mathrm{C}$ terminus of drebrin and an additional Bam HI site at its $3^{\prime}$ end. The products were digested with XhoI and BamHI and cloned into $X h o I / B a m H I$ digested EGFP-C1 vector. The drebrins insert was generated by combining upstream and downstream fragments and was fused to GFP by cloning into XhoI/Bam HI site of EGFP-C1 vector. The upstream fragment was an $X h o I / K p n I$ digested PCR product that was amplified using the $5^{\prime}$ primer described above and a primer containing antisense sequence of drebrin cDNA encoding amino acid residues 226-232, followed by an additional KpnI site (AAAGGTACCATCTGCTGCTGCTCCCGCTCTCG). The downstream fragment was a $K p n \mathrm{I} /$ Bam HI digested PCR product that was amplified with the $3^{\prime}$ primer described above and a primer containing a sense cDNA sequence encoding amino acid residues 301-307 of drebrin after an additional KpnI site (AAAGGTACCGGCTTCAGCCTCTGGTGGCA). Combining these two fragments created an extra sequence encoding Gly-Thr at the junction.

Western blotting. For Western blotting, proteins of $35 \mathrm{~mm}$ dish cultures of $\mathrm{CHO}$ cells were extracted with SDS sample buffer, and 1/20 aliquots of them were separated by polyacrylamide SDS-gel electrophoresis. They were blotted onto an Immobilon Transfer Membrane (Millipore, Bedford, MA). The membranes were incubated in skim milk for $>4 \mathrm{hr}$ and subsequently with a rat monoclonal anti-GFP antibody (generously donated by Dr. Shinobu Fujita, Mitsubishi Kagaku Lifescience Institute, Japan) or a mouse monoclonal anti-drebrin antibody for $1 \mathrm{hr}$. After washing in PBS for $30 \mathrm{~min}$, they were incubated with the secondary antibody (biotin-conjugated goat IgG against rat or mouse IgG; Vector Laboratories, Burlingame, CA) for $1 \mathrm{hr}$ and washed again. Immunore- action was visualized with an avidin-biotinylated horseradish peroxidase complex (Vectastain kit; Vector Laboratories) and diaminobenzidine.

Confocal microscopy and measurements. Confocal microscopic images were obtained with a Bio-Rad (Hercules, CA) MRC600. For observation of dendritic spines, 10-20 serial images of $0.8 \mu \mathrm{m}$ thickness were projected onto one plane. Spine length and density were measured in the projected images using NIH Image software. Simple averages of 25-200 measurements of spine lengths in each neuron were calculated and ascribed to the neuron as its average spine length. Spine density was measured from images spanning greater than $50 \mu \mathrm{m}$ of a dendrite. Twelve GFP-expressing and 16 GFP-drebrin-expressing neurons were analyzed and statistically compared.

\section{RESULTS \\ Colocalization of drebrin and actin filaments at spines in primary cultures of cortical neurons}

In high-density primary culture systems, cortical neurons begin to form synapses at 1 week in vitro (Ichikawa et al., 1993; Hayashi et al., 1998), and synapses undergo morphological maturation for several weeks thereafter (Ichikawa et al., 1993; Papa et al., 1995). Actin filaments are known to be concentrated at spines of mature neurons (Fifkova and Delay, 1982; Matus et al., 1982). To confirm the localization of actin filaments at spines in our 3-weekold cultures, we stained cultures with rhodamine-phalloidin. Actin filaments were detected as scattered dots (Fig. 1A,D,G). Confocal laser microscopic observation of double staining with an anti-synaptophysin antibody and phalloidin revealed that most of the phalloidin-positive dots were closely associated with synaptophysin-positive dots, indicating that they are postsynaptic (Fig. 1A). When we observed an isolated dendrite at an uncrowded area of the culture, we could identify the phalloidinpositive dots at the tip of spines that were seen to extend from the dendrite (Fig. 1B). Rhodamine-phalloidin also weakly stained the submembranous region of neuron cell bodies and dendrites (Fig. 1C). Next, we examined the colocalization of drebrin and actin filaments in culture, double staining cells with rhodaminephalloidin and an anti-drebrin antibody. Drebrin was detected as scattered dots (Fig. 1E) and most of these dots (191 of 200 dots, $95.5 \%$ ) were also positive for rhodamine-phalloidin (Fig. $1 F$ ), indicating that drebrin is associated with actin filaments at spines. Although we observed that some phalloidin-positive dots (11 of 200 dots, $5.5 \%$ ) were not positive for drebrin (Fig. $1 G-I$, arrows), we could not determine whether they were really in spines. We also noted that drebrin was hardly detected at cell membranes and neurites (Fig. 1D-F, arrowheads, double arrowheads).

\section{Actin binding of GFP-drebrin in $\mathrm{CHO}$ cells}

We recently located the actin-binding domain of drebrin at its central region. The domain was identified by analyzing the association of mutant drebrin with actin filaments in $\mathrm{CHO}$ cells transfected with various mutated drebrin cDNAs and by using a cosedimentation assay of chymotryptic fragments of drebrin with purified actin filaments (our unpublished observations). The mutant drebrin cDNAs that do not have the actin-binding domain did not bind to actin filaments and did not remodel actin filaments in $\mathrm{CHO}$ cells, and introducing this domain into $\mathrm{CHO}$ cells resulted in binding of this peptide with actin filaments and remodeling of actin filaments. We suppose that this domain is the only region that is responsible for the actin-binding and actinremodeling activities of drebrin.

In the present study, two chimeric cDNAs carrying GFP cDNA and drebrin A cDNA, with and without the actin-binding domain, were constructed (Fig. 2). One, named GFP-drebrin, has the full-length sequence of rat drebrin A. The other, named GFP- 

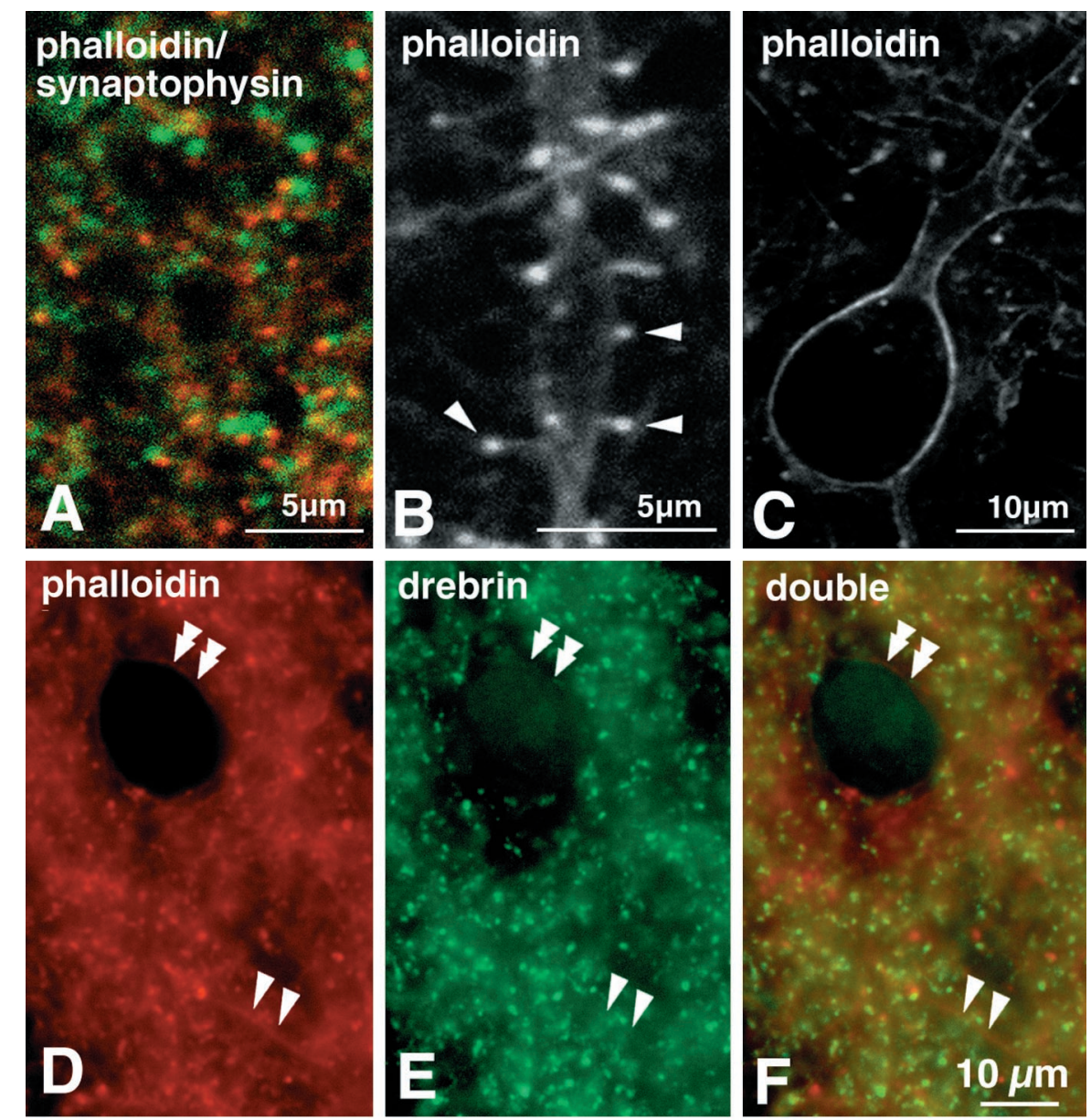

Figure 1. Colocalization of drebrin with actin filaments at spines in primary cultures of cortical neurons at 3 weeks in vitro. $A$, Confocal observation of neurons that were double-stained with rhodamine-phalloidin and an anti-synaptophysin antibody. Most phalloidin-positive dots are closely associated with synaptophysin-positive dots. $B$, Confocal observation of a dendrite at an uncrowded area of the culture stained with rhodamine-phalloidin. Phalloidin-positive dots are revealed to be spine heads (arrowheads). $C$, Confocal observation of the cell body of a neuron stained with rhodamine-phalloidin. $D-I$, Neurons were double-stained with rhodamine-phalloidin and an anti-drebrin antibody. Yellowish dots in the composite pictures demonstrate the colocalization of drebrin and actin filaments. $D-F$ are observed with an epifluorescent microscope and $G-I$ with a confocal microscope. Single arrowheads in $D-F$ indicate dendritic shafts, and double arrowheads indicate cell soma that was identified using Nomarski optics. The dendritic shafts and cell membranes were weakly stained with rhodamine-phalloidin but hardly with an anti-drebrin antibody. Arrow in $I$ indicates a rare instance in which a phalloidinpositive dot was negative for drebrin.
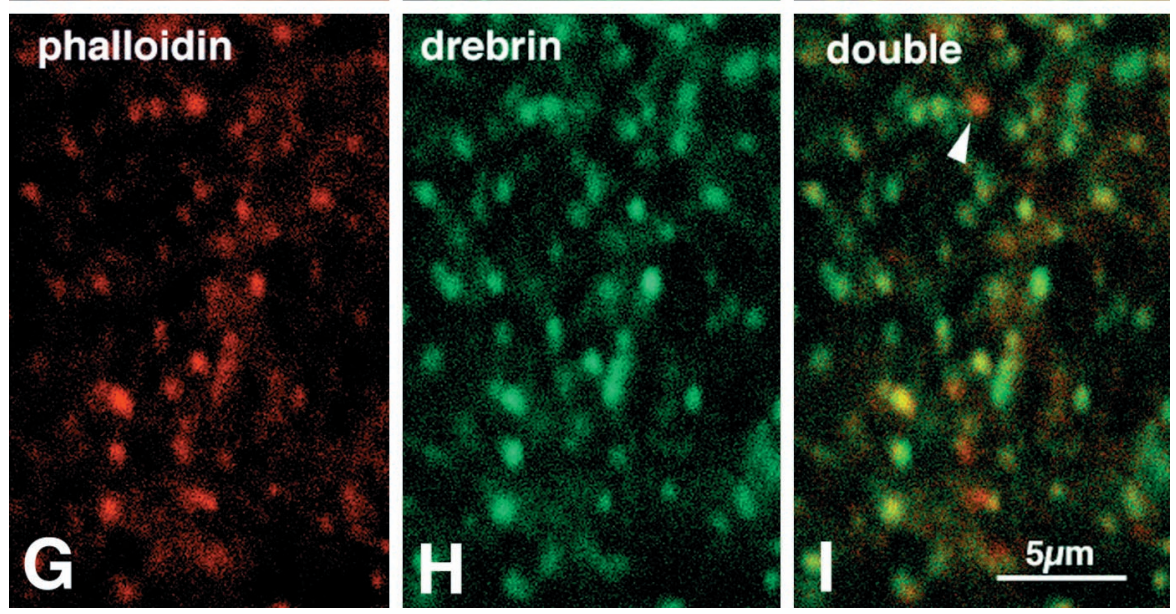

drebrin $\Delta$, has a drebrin A sequence that carries a deletion in the actin-binding domain (amino acid residues 233-300). To test the ability of these chimeric proteins to bind to actin filaments, we transfected $\mathrm{CHO}$ cells with these cDNAs and examined the association of these chimeric proteins with actin filaments. In all CHO cells expressing GFP-drebrin, fluorescence was colocalized with actin filaments labeled with rhodamine-phalloidin. Over $50 \%$ of the cells exhibited remodeling of actin filaments or a drastic change in cell shape as shown in Figure $3 A, C$. The morphology of GFP-drebrin-expressing cells was virtually identical to that described in our previous report (Shirao et al., 1994) in which drebrin cDNA was introduced into $\mathrm{CHO}$ cells without tag, indicating that GFP tagging did not disturb the actin-binding and actin-remodeling activity of drebrin. In contrast, GFPdrebrin $\Delta$ was found to be distributed diffusely within cells, and only a very small proportion was colocalized with actin filaments. GFP-drebrin $\Delta$-expressing cells did not exhibit remodeling of actin filaments or change in cell shape (Fig. $3 B, D$ ).

Full-length expression of chimeric polypeptides in $\mathrm{CHO}$ cells was confirmed by Western blot analysis (Fig. $3 E$ ). Transfection of unfused GFP cDNA resulted in a single band at $\sim 35 \mathrm{kDa}$ that reacted with an anti-GFP antibody (Fig. 3E, left). Transfection with GFP-drebrin cDNA produced a band at $\sim 170 \mathrm{kDa}$, which is $\sim 40 \mathrm{kDa}$ larger than the molecular weight obtained previously for 


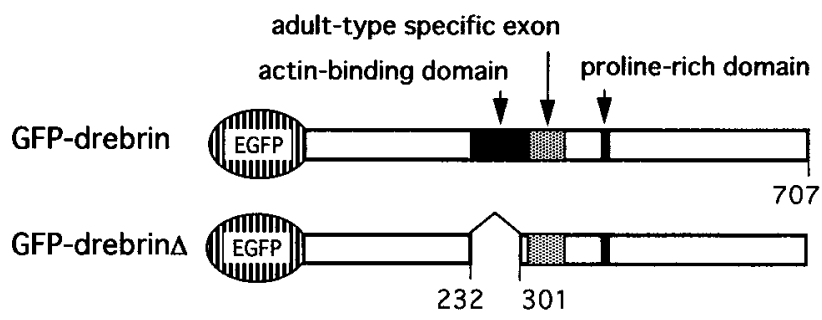

Figure 2. Schematic representation of cDNA constructs. Rat drebrin A with (top) or without (bottom) an actin-binding region was linked to the $\mathrm{C}$ terminus of EGFP. Hatched boxes indicate the adult-type-specific exon of drebrin. The proline-rich domain indicated is supposed to be the site of profilin binding. Numbers indicate the amino acid residue numbers of drebrin.

drebrin A. The anti-GFP positive band of GFP-drebrin $\Delta$ transfected cells migrated slightly faster than that of GFPdrebrin-transfected cells. Both bands were also detected with an anti-drebrin antibody (Fig. $3 E$, right). The intensity of the two bands was nearly equal, suggesting that the mutation does not alter the level of expression.

\section{Localization of GFP-drebrin in neurons}

We transfected a dense culture of dissociated neurons from rat cerebral cortex with the cDNAs described above. Transfection was performed on day 7 in vitro to minimize the possible effects of drebrin overexpression on neurite elongation. Figure $4 A$ shows the morphology of a neuron at this stage. The neuron had already developed principal morphology of dendritic arborization while some dendritic growth cones were still apparent (Fig. 4A, arrowheads). Dendritic filopodia were also visible (Fig. 4A, arrows). The transfected cultures were fixed at 3 weeks in vitro when the synapses were sufficiently mature (Ichikawa et al., 1993; Papa et al., 1995; Hayashi et al., 1998). In the GFP-transfected culture, intense green fluorescence was observed within cell somata and dendritic shafts (Fig. 4A). In the case of GFP-drebrin, the fluorescence was seen as dots that were associated with dendrites but was weak in dendritic shafts (Fig. 4B). Confocal microscopy revealed that GFP-drebrin was localized at spines (Fig. 5A-D). The fluorescence in dendritic shafts was weak compared with that at spines. In contrast with GFP-drebrin, high levels of GFP (Fig. $5 E$ ) and GFP-drebrin $\Delta$ (Fig. $5 F$ ) were detected in dendritic shafts. Failure of GFP-drebrin $\Delta$ to accumulate at spines indicates that specific localization of drebrin at spines requires its actinbinding domain.

To confirm that the spines labeled with GFP-drebrin were actually postsynaptic, we stained the GFP-drebrin-expressing neurons with an anti-synaptophysin antibody (Fig. 6). Because this was a dense culture, many spots were stained with the anti-synaptophysin antibody (Fig. 6B), and some of them were closely associated with the heads of GFP-drebrin-labeled spines (Fig. 6C, large arrowheads).

\section{Morphological changes of the spines labeled with GFP-drebrin}

Some spines labeled with GFP-drebrin were markedly longer than any of the GFP-labeled spines we observed. To assess statistically the effect of drebrin overexpression on spine length, the lengths of 923 spines of 12 GFP-expressing neurons and of 1193 spines of 16 GFP-drebrin-expressing neurons were measured from confocal microscopic images. The distribution of these measurements is shown in Figure $7 A$. We sometimes observed extraordinarily long spines, over $5 \mu \mathrm{m}$, in GFP-drebrinexpressing neurons. Examples of such long spines are shown in Figure $6 A$ (small arrowheads). These long spines were different from dendritic filopodia, which are the precursors of spines and
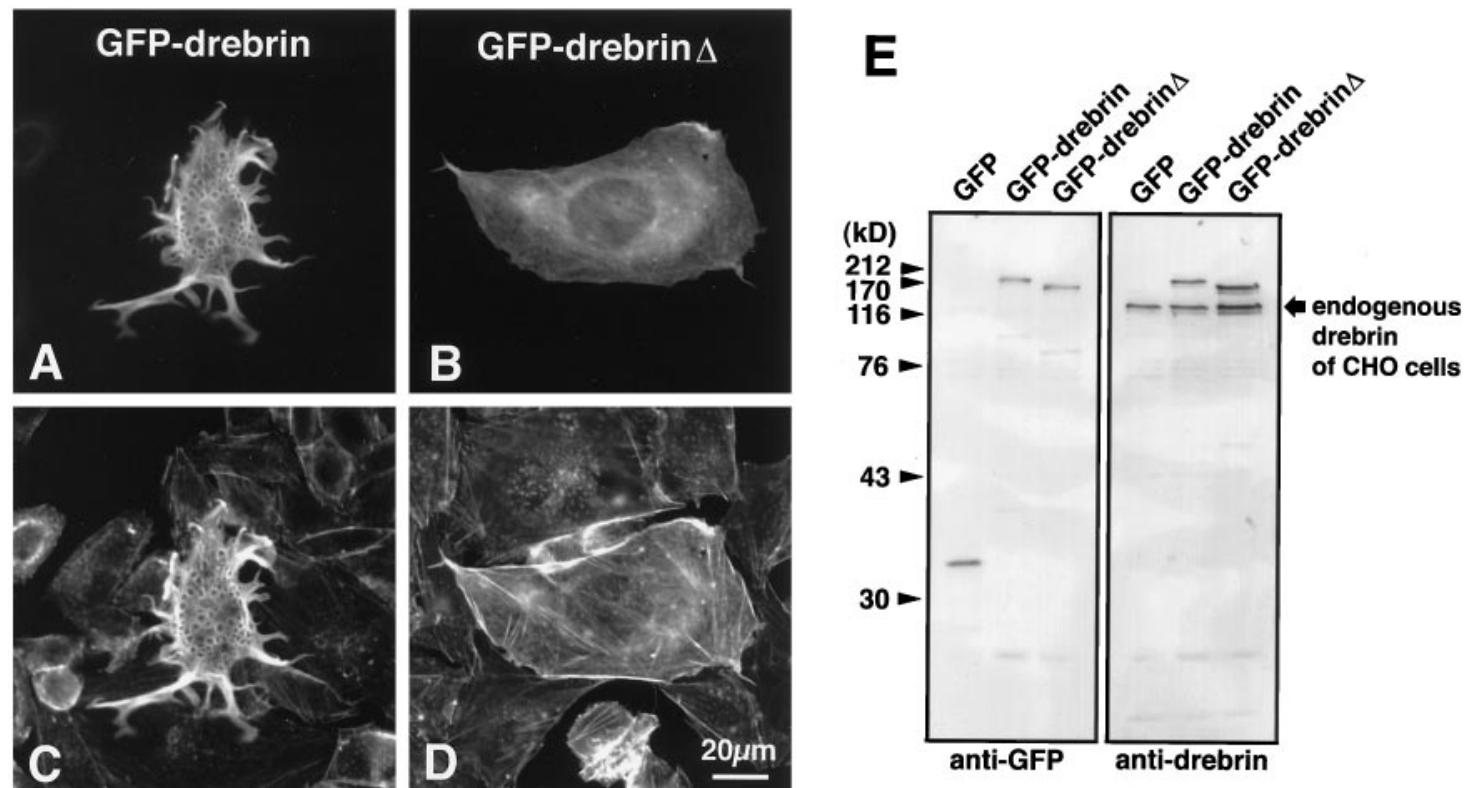

Figure 3. Fluorescence microscopic images and Western blot analysis of CHO cells expressing GFP-fusion proteins. Cells were transfected with GFP-drebrin cDNA $(A, C)$ and GFP-drebrin $\Delta$ cDNA $(B, D)$. They were stained with rhodamine-phalloidin to visualize actin filaments and observed using FITC $(A, B)$ and rhodamine $(C, D)$ filter sets. Note that GFP-drebrin bound to actin filaments and caused remodeling of actin filaments, whereas a very small proportion of GFP-drebrin $\Delta$ was colocalized with actin filaments, and no remodeling of actin filaments was observed in GFP-drebrin $\Delta$ expressing cells. Western blotting of CHO cells transfected with cDNAs for GFP, GFP-drebrin, or GFP-drebrin $\Delta$ is shown in E. Membranes were immunostained with an anti-GFP antibody (left) and an anti-drebrin antibody (right). The position of the endogenous drebrin of CHO cells (embryonic type) is indicated with an arrow. 

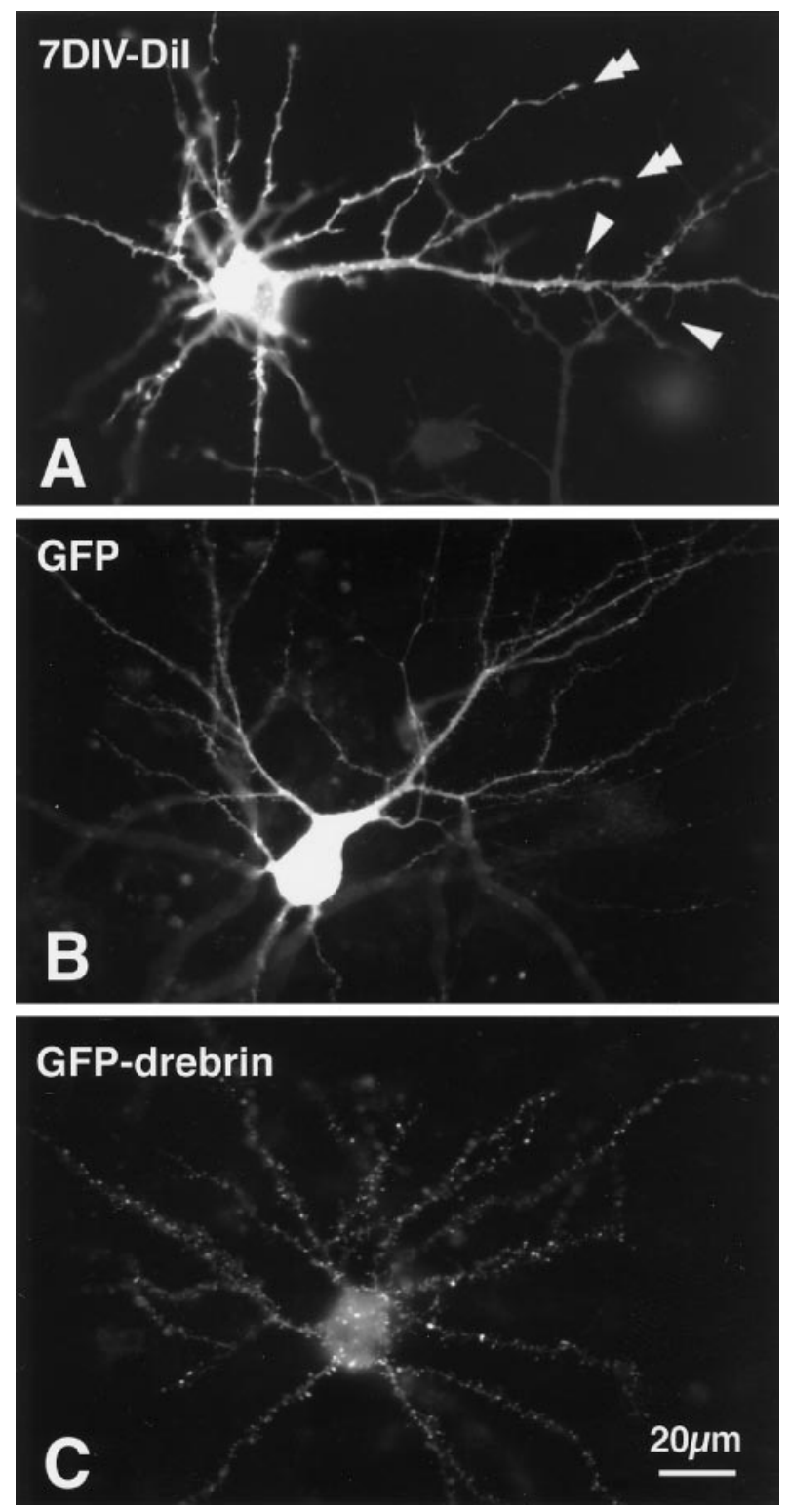

Figure 4. Fluorescence microscopic images of neurons before and 2 weeks after transfection. $A$, A neuron at $7 \mathrm{~d}$ in vitro was labeled with DiI to show morphology before transfection. Arrowheads indicate dendritic filopodia, and double arrowheads indicate dendritic growth cones. Note that principal morphology of dendritic arborization has been already developed. $B$, A GFP-transfected neuron at 3 weeks in vitro. Fluorescence was seen in cell bodies and dendritic shafts. $C$, A GFP-drebrin-transfected neuron at 3 weeks in vitro. Fine dots are seen around the dendrites.

are relatively long (Papa et al., 1995; Dailey and Smith, 1996; Ziv and Smith, 1996), because they were actually associated with axonal terminals (Fig. 6C).

The average spine length from each individual neuron was calculated, and the distribution of these values is shown in Figure $7 B$. The average value from neurons expressing GFP-drebrin were significantly ( $p<0.0001 ; t$ test) longer than that in GFPexpressing neurons (Fig. 7B). To exclude the possibility that we underestimated the spine length of GFP-expressing neurons as a result of incomplete diff usion of GFP into spine heads, we measured spine length from neurons labeled with DiI (Fig. 7B, dashed column). The average spine length of DiI-labeled neurons was not significantly different from that of GFP-expressing neurons and was significantly shorter than that of GFP-drebrin-expressing neurons.

We also compared spine densities along dendrites of GFPexpressing neurons and those of GFP-drebrin-expressing neurons (Fig. 7C). The spine density in GFP-expressing neurons was $0.76 \pm 0.13$ (per $1 \mu \mathrm{m})$, whereas that in GFP-drebrin-expressing neurons was $0.96 \pm 0.25$ (mean \pm SD). The difference between the two was not significant $(p>0.01)$.

\section{DISCUSSION}

In this study, we examined our hypothesis that drebrin is one of the regulators of spine shape. First, we demonstrated that drebrin and actin filaments are colocalized at spines in long-term primary culture of cortical neurons. Second, we demonstrated that GFPtagged drebrin A accumulated at dendritic spines when introduced into cultured neurons and that this accumulation required actin binding. Finally, we examined the effect of GFP-drebrin on spine shape and found that exogenously expressed drebrin A caused spine elongation.

\section{Actin-dependent localization of drebrin at the spines}

We confirmed that drebrin is localized specifically at spines in our culture system, as in the previous observation in vivo (Shirao et al., 1987; Hayashi et al., 1996). Drebrin was colocalized with actin filaments, which are concentrated at spines. Although the previous observation did not exclude the possibility that drebrin is expressed only in a subset of spines, the present data, that drebrin was expressed at almost all spines in cultured neurons, indicates that drebrin expression is closely associated with the presence of spines.

Drebrin was concentrated in spines but not detected in dendritic shafts. We assume that the binding sites of actin filaments in dendritic shafts are occupied by other actin-binding proteins, such as microtubule-associated protein 2 (MAP2) or tropomyosin. Actually, MAP2, which is highly expressed at dendritic shafts and has an activity to bind to actin filaments, is nearly absent at spines (Kaech et al., 1997). Thus, actin filaments in spines must have a different dynamic nature from those in dendritic shafts. In fact, differences in the stability of actin filaments against actindepolymerizing reagents have been reported between actin filaments in spines and those in dendritic shafts or cell bodies (Allison et al., 1998). In neuroblastoma cells, actin filaments with drebrin were stable against cytochalasin D, but those without drebrin were not (Asada et al., 1994). Therefore, specific accumulation of drebrin at the spines may be responsible for the cytochalasin-resistance of actin filaments at spines.

GFP-drebrin accumulated at dendritic spines, whereas GFPdrebrin $\Delta$, which lacks actin-binding activity, did not. This provides a further and critical confirmation that drebrin binds to actin filaments at spines. It also indicates that the specific localization of drebrin at spines is dependent on the presence of actin filaments there.

Little is known about the mechanisms of actin accumulation at spines, although much information has been gathered about protein complexes that constitute postsynaptic densities (for review, see Craven and Bredt, 1998). $\alpha$-Actinin is known to be concentrated at spines and to bind to NMDA receptors. However, the NMDA receptor is not likely to be the primary anchor for $\alpha$-actinin and actin filaments, because $\alpha$-actinin clusters at spines before APV-dependent clustering of NMDA receptors in cultured hippocampal neurons (Rao and Craig, 1997). Actin polymerization at spines is likely to be regulated by small GTPases, as 

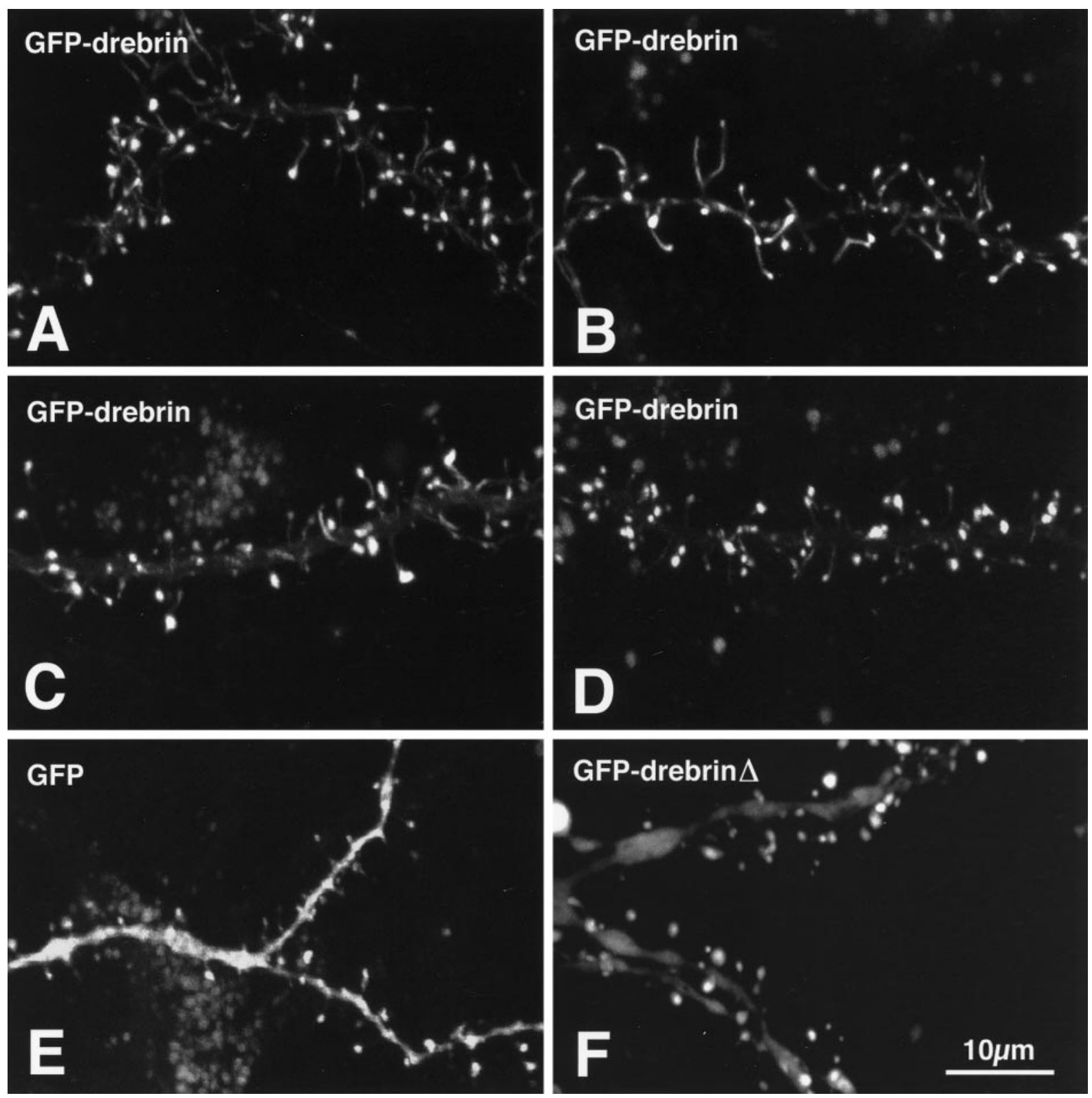

Figure 5. Confocal microscopic images of neurons expressing GFP-drebrin $(A-D)$, GFP $(E)$, and GFP-drebrin $\Delta(F)$. $A-D$ were taken from four different neurons. Fluorescence was concentrated at spines of GFP-drebrin-expressing neurons. In GFP- and GFP-drebrin $\Delta$-expressing neurons, considerable fluorescence was observed in dendritic shafts.

suggested by investigators studying transgenic mice that express constitutively active Rac1. In these mice, spines of Purkinje neurons were reduced in size but increased in number (Luo et al., 1996).

The slight increase in spine density after drebrin overexpression was not significant, and this increase can be explained by the possibilities that the elongation of GFP-drebrin-labeled spines makes them easy to detect and that fluorescence of dendritic shafts in GFP-expressing neurons makes spines behind them difficult to detect. Because spine density increases until the end of week 3 in vitro (Papa et al., 1995), the lack of a significant change in spine density in the presence of exogenous drebrin during the second and third weeks in vitro suggests that drebrin did not induce spine formation. However, we cannot rule out the possibility that spine precursors are generated during the first week of cultivation and that the drebrin transfection on day 7 in this study was too late to exert an effect on spine formation.

\section{Drebrin-related elongation of spines}

Many mechanisms are possibly responsible for the elongation of spines after overexpression of drebrin. Because we investigated the morphology of spines 2 weeks after transfection, it is possible that the elongation was a secondary effect of changes in synaptic activity or in activity of other proteins that was influenced by drebrin. However, our data that drebrin binds to actin filaments at spines and our knowledge that actin filaments are the primary modulator of spine shape suggest that drebrin directly acts on actin filaments at spines to cause morphological change.

We reported previously that drebrin inhibits the actin-binding activities of tropomyosin and $\alpha$-actinin (Ishikawa et al., 1994), 

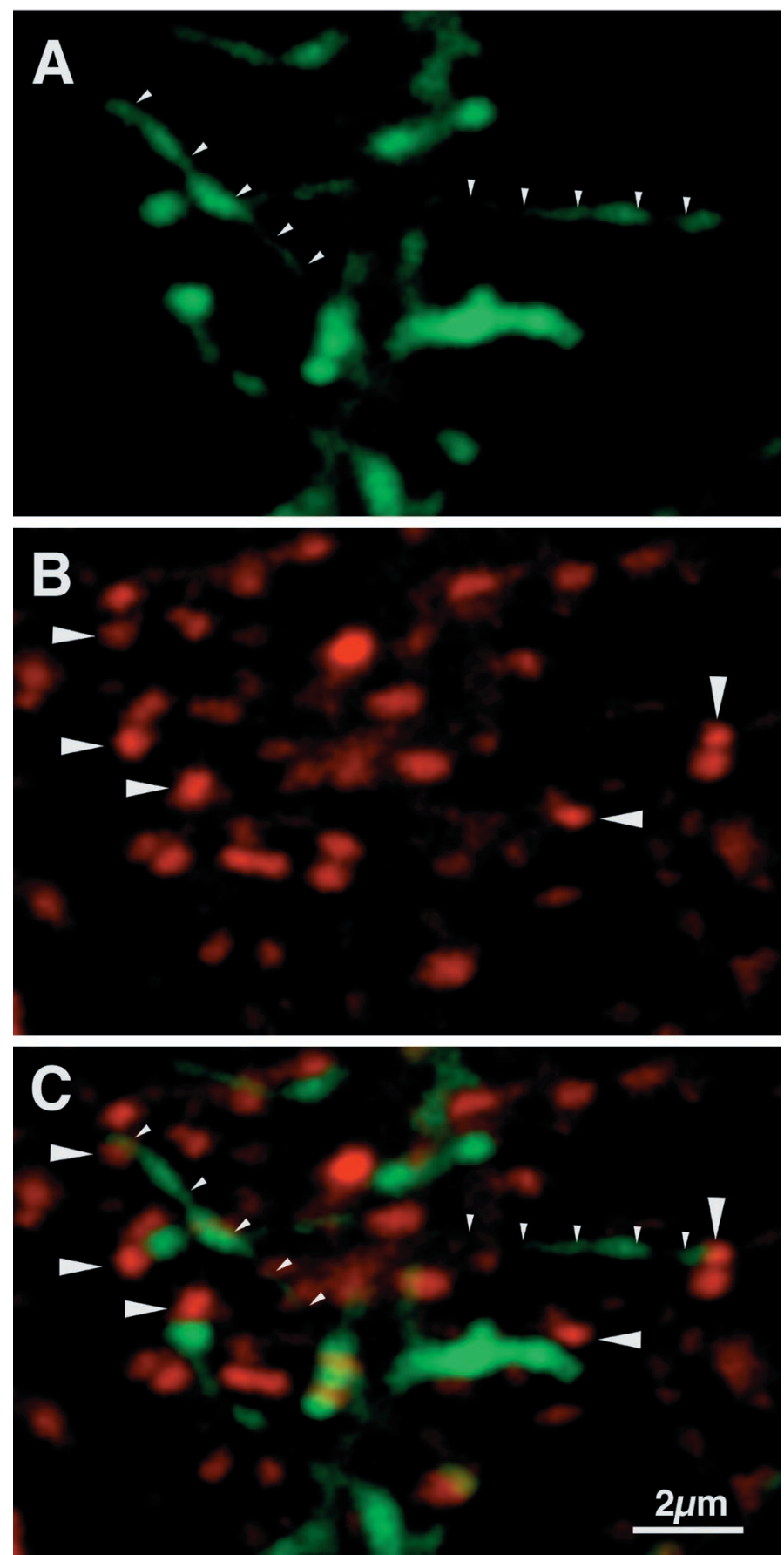

Figure 6. The spines labeled with GFP-drebrin were associated with axonal terminals. A GFP-drebrin-expressing neuron $(A)$ was immunostained with an anti-synaptophysin antibody $(B)$. $C$ shows the composite. The small arrowheads indicate two spines that are $\sim 5 \mu \mathrm{m}$ long. The large arrowheads indicate synaptophysin-stained presynaptic terminals that are attached to the spine heads labeled with GFP-drebrin.

both of which are known to stabilize actin filaments by protecting them from severance caused by gelsolin (Ishikawa et al., 1989a,b) and actin-depolymerizing factor (Bernstein and Bamburg, 1982). The inhibition occurs at concentrations below $1 \mu \mathrm{M}$ drebrin, which we suppose is a possible in vivo concentration of drebrin at spines. Localization of drebrin at spines, thus, may result in the maintenance of actin filaments in a dynamic state, which enables them to be reactive to synaptic stimuli (Hayashi et al., 1996).
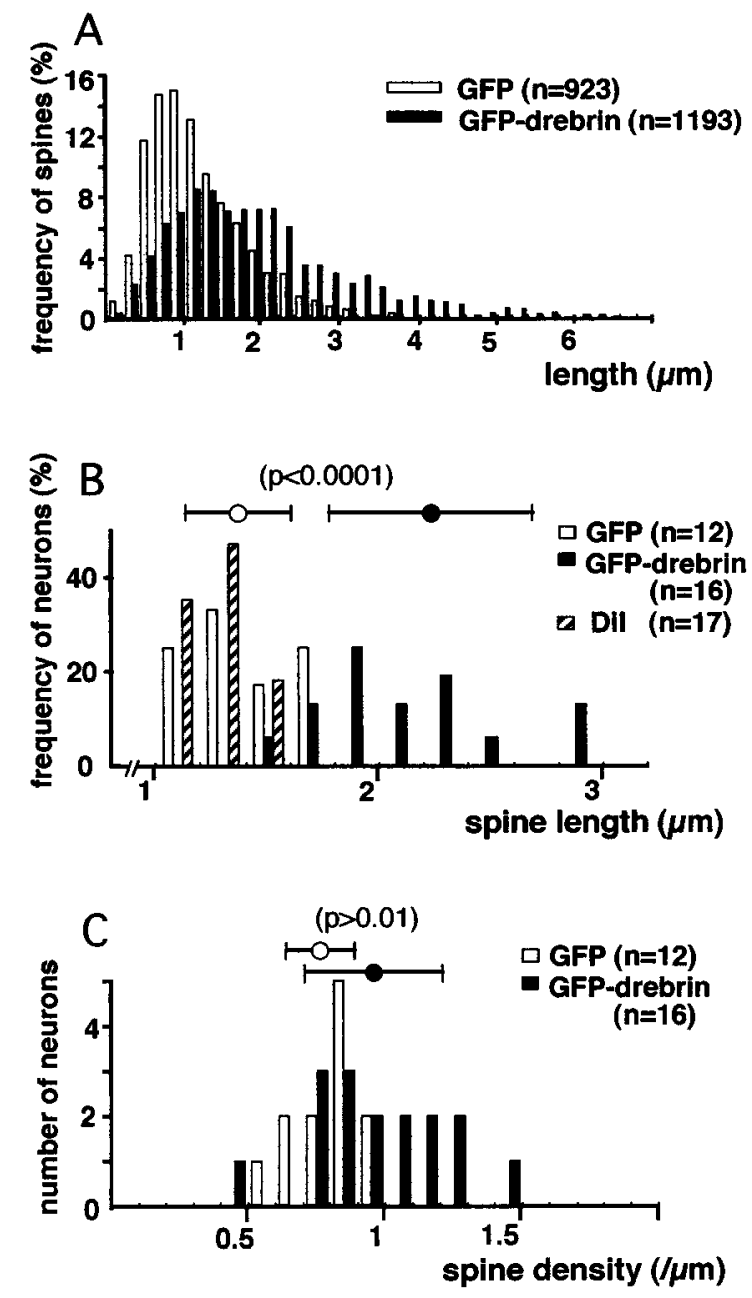

Figure 7. Histograms showing the distribution of length and density of the spines labeled with GFP or GFP-drebrin. A, The length of 50-200 spines was measured in each neuron, giving measurements of 923 in GFP-expressing neurons and 1193 in GFP-drebrin-expressing neurons. $B$, The average length of spines was calculated for each neuron. The values of 16 GFP-expressing neurons, 12 GFP-drebrin-expressing neurons, and 17 DiI-labeled neurons are represented in a histogram. $C$, Spine densities of 16 GFP-expressing neurons and 12 GFP-drebrin-expressing neurons are shown. Error bars show SDs.

Consistent with this idea, the dynamic motility of actin within spines was beautifully demonstrated by Fischer et al. (1998), and this short time-scale motility was revealed to be dependent on the dynamics of actin filaments themselves. When expressed at physiologically impossible high levels as in this study, drebrin might hyperdestabilize actin filaments in spines and alter spine shape.

The inhibitory effect of drebrin on actin-myosin interaction (Hayashi et al., 1996) may also be responsible for the elongation of spines in GFP-drebrin-expressing neurons. We showed previously that drebrin reduced the sliding velocity of actin filaments on immobilized myosin and inhibited actin-based ATPase activity of myosin (Hayashi et al., 1996). GFP-drebrin in spines may reduce the contractile force of actomyosin and thereby inhibit spines retraction. Retraction of long dendritic filopodia into mushroom-shaped spines is a normal event during maturation of the spines (Papa et al., 1995; Dailey and Smith, 1996). Interestingly, it has been reported that filopodia of growth cones elongate when myosin activity is inhibited (Lin et al., 1996). In this case, 
proximally oriented flow of actin filaments is inhibited, and polymerization at the tip of the filopodia results in their abnormal elongation.

Drebrin has been revealed to bind to profilin, an actin-binding protein that stimulates actin polymerization (Mammoto et al., 1998). Among the well known profilin-binding proteins are vasodilator-stimulated phosphoprotein and mena (Gertler et al., 1996). These proteins bind to profilin via their proline-rich sequence and stimulate actin polymerization. Drebrin also has a proline-rich sequence at 410-419 amino acids (see Fig. 2), and this may be responsible for the reported profilin binding. It is possible that profilin is recruited into spines via drebrin, stimulates actin polymerization, and thereby causes elongation of the spines. However, the physiological function of profilin binding of drebrin is not yet clear because this proline-rich sequence is not preserved in chicken drebrin.

Other actin-binding proteins are also known to be specifically localized at spines. $\alpha$-Actinin is localized at spines and is thought to cross-link NMDA receptors and actin filaments (Wyszynski et al., 1997). Synaptopodin is also an actin-associated protein known to be localized at spines (Mundel et al., 1997). There is no evidence, however, that these proteins are involved in the regulation of spine shape. Effects of overexpression or misexpression of any of these proteins on spine shape have not been studied. We present here direct evidence that drebrin influences spine shape. Our data are consistent with the idea that drebrin is responsible for the dynamic remodeling of actin filaments in spines and influences spine shape.

\section{REFERENCES}

Allison DW, Gelfand VI, Spector I, Craig AM (1998) Role of actin in anchoring postsynaptic receptors in cultured hippocampal neurons: differential attachment of NMDA versus AMPA receptors. J Neurosci 18:2423-2436.

Asada H, Uyemura K, Shirao T (1994) Actin-binding protein, drebrin, accumulates in submembranous regions in parallel with neuronal differentiation. J Neurosci Res 38:149-159.

Bernstein BW, Bamburg JR (1982) Tropomyosin binding to F-actin protects the F-actin from disassembly by brain actin-depolymerizing factor (ADF). Cell Motil 2:1-8.

Craven SE, Bredt DS (1998) PDZ proteins organize synaptic signaling pathways. Cell 93:495-498.

Dailey ME, Smith SJ (1996) The dynamics of dendritic structure in developing hippocampal slices. J Neurosci 14:1060-1078.

Edwards FA (1995) LTP — a structural model to explain the inconsistencies. Trends Neurosci 18:250-255.

Fifkova E, Delay RJ (1982) Cytoplasmic actin in neuronal processes as a possible mediator of synaptic plasticity. J Cell Biol 95:345-350.

Fischer M, Kaech S, Knutti D, Matus A (1998) Rapid actin-based plasticity in dendritic spines. Neuron 20:847-854.

Gertler FB, Niebuhr K, Reinhard N, Wehland J, Soriano P (1996) Mena, a relative of VASP and Drosophila enabled, is implicated in the control of microfilament dynamics. Cell 87:227-239.

Gold JI, Bear MF (1994) A model of dendritic spine $\mathrm{Ca}^{2+}$ concentration exploring possible bases for a sliding synaptic modification threshold. Proc Natl Acad Sci USA 91:3941-3945.

Harris KM, Kater SB (1994) Dendritic spines: cellular specializations imparting both stability and flexibility to synaptic function. Annu Rev Neurosci 17:341-371.

Hayashi K, Ishikawa R, Ye L-H, He X-L, Takata K, Kohama K, Shirao $\mathrm{T}$ (1996) Modulatory role of drebrin on the cytoskeleton within dendritic spines in the rat cerebral cortex. J Neurosci 16:7161-7170.

Hayashi K, Suzuki K, Shirao T (1998) Rapid conversion of drebrin isoforms during synapse formation in primary culture of cortical neurons. Dev Brain Res 111:137-141.

Hosokawa T, Rusakov DA, Bliss TVP, Fine A (1995) Repeated confocal imaging of individual dendritic spines in the living hippocampal slice: evidence for changes in length and orientation associated with chemically induced LTP. J Neurosci 15:5560-5573.

Ichikawa M, Muramoto K, Kobayashi K, Kawahara M, Kuroda Y (1993) Formation and maturation of synapses in primary cultures of rat cerebral cortical cells: an electron microscopic study. Neurosci Res 16:95-103.

Ishikawa R, Yamashiro S, Matsumura F (1989a) Differential modulation of actin-severing activity of gelsolin by multiple isoforms of cultured rat cell tropomyosin: potentiation of protective ability of tropomyosins by 83-kDa nonmuscle caldesmon. J Biol Chem 264:7490-7497.

Ishikawa R, Yamashiro S, Matsumura F (1989b) Annealing of gelsolinsevered actin fragment by tropomyosin in the presence of $\mathrm{Ca}^{2+}$ : potentiation of annealing process by caldesmon. $\mathrm{J}$ Biol Chem 264:16764-16770.

Ishikawa R, Hayashi K, Shirao T, Xue Y, Takagi T, Sasaki Y, Kohama K (1994) Drebrin, a development-associated brain protein from rat embryo, causes the dissociation of tropomyosin from actin filaments. J Biol Chem 47:29928-29933.

Kaech S, Fischer M, Doll T, Matus A (1997) Isoform specificity in the relationship of actin to dendritic spines. J Neurosci 17:9565-9572.

Koch C, Zador A (1993) The function of dendritic spines: devices subserving biochemical rather than electrical compartmentalization. J Neurosci 13:413-422.

Lin CH, Espreafico EM, Mooseker MS, Forscher P (1996) Myosin drives retrograde F-actin flow in neuronal growth cones. Neuron 16:796-782.

Luo L, Hensch TK, Ackerman L, Barbel S, Jan LY, Jan YN (1996) Differential effects of the Rac GTPase on Purkinje cell axons and dendritic trunks and spines. Nature 379:837-840.

Mammoto A, Sasaki T, Asakura T, Hotta I, Imamura H, Takahashi K, Matsuura Y, Shirao T, Takai Y (1998) Interactions of drebrin and gephyrin with profilin. Biochem Biophys Res Commun 243:86-89.

Matus A, Ackermann M, Pehling G, Byers HR, Fujiwara K (1982) High actin concentrations in brain dendritic spines and postsynaptic densities. Proc Natl Acad Sci USA 79:7590-7594.

Mundel P, Heid HW, Mundel TM, Kruger M, Reiser J, Kriz W (1997) Synaptopodin: an actin-associated protein in telencephalic dendrites and renal podocytes. J Cell Biol 139:193-204.

Obata K, Nishiye H, Fujita S, Shirao T, Inoue H, Uchizono K (1986) Identification of a synaptic vesicle-specific 38,000-dalton protein by monoclonal antibodies. Brain Res 375:37-48.

Papa M, Bundman MC, Greenberger V, Segal M (1995) Morphological analysis of dendritic spine development in primary cultures of hippocampal neurons. J Neurosci 15:1-11.

Rao A, Craig M (1997) Activity regulates the synaptic localization of the NMDA receptor in hippocampal neurons. Neuron 19:801-812.

Sasaki Y, Hayashi K, Shirao T, Ishikawa R, Kohama K (1996) Inhibition by drebrin of the actin-bundling activity of brain fascin, a protein localized in filopodia of growth cones. J Neurochem 66:980-988.

Shirao T (1995) The roles of microfilament-associated proteins, drebrins, in brain morphogenesis: a review. J Biochem 117:231-236.

Shirao T, Inoue HK, Kano Y, Obata K (1987) Localization of a developmentally regulated neuron-specific protein S54 in dendrites as revealed by immunoelectron microscopy. Brain Res 413:374-378.

Shirao T, Kojima N, Obata K (1992) Cloning of drebrin A and induction of neurite-like processes in drebrin-transfected cells. NeuroReport 3:109-112.

Shirao T, Hayashi K, Ishikawa R, Isa K, Asada H, Ikeda K, Uyemura K (1994) Formation of thick curving bundles of actin by drebrin A expressed in fibroblasts. Exp Cell Res 215:145-153.

Wyszynski M, Lin J, Rao A, Nigh E, Beggs AH, Craig AM, Sheng M (1997) Competitive binding of $\alpha$-actinin and calmodulin to the NMDA receptor. Nature 385:439-442.

Ziv NE, Smith SJ (1996) Evidence for a role of dendritic filopodia in synaptogenesis and spine formation. Neuron 17:91-102. 